\title{
A real time non-invasive cholesterol monitoring system.
}

\author{
Usman Umar ${ }^{1,2, *}$, Syafruddin Syarif ${ }^{3}$, Ingrid Nurtanio ${ }^{4}$ and Indrabayu ${ }^{4}$ \\ ${ }^{1}$ Doctoral Student, Electrical Engineering Department, Hasanuddin University Makassar, Indonesia \\ ${ }^{2}$ Department of Electro Medical Technology, Poltekkes Muhammadiyah Makassar, Indonesia \\ ${ }^{3}$ Electrical Engineering Department, Hasanuddin University Makassar, Indonesia \\ ${ }^{4}$ Informatics Department, Hasanuddin University Makassar, Indonesia
}

\begin{abstract}
Hypercholesterolemia causes cardiovascular disease which is a disorder of the heart and blood vessels, one of the most significant causes of death in the world, and this needs to be anticipated by monitoring blood cholesterol levels regularly. The current method of monitoring blood cholesterol levels is using invasive technique by collecting blood samples. A simple device is needed to measure blood cholesterol levels that can be done without collecting blood samples. This study aims to develop a noninvasive technique for monitoring blood cholesterol levels using sensors utilizing infrared light absorption in body tissues, in order to simplify measuring blood cholesterol levels regularly for patients with hypercholesterolemia. The application of a non-invasive technique focused on developing a total blood cholesterol monitoring device using an infrared sensor with IR LED - 940nm wavelength as a transmitter. A photodiode was used as a detector with the wavelength range of 400-1100 nm and a microcontroller as the minimum system for controlling the value of the output voltage in the form of digital data and then converted onto total blood cholesterol. The measurement results using non-invasive technique was compared to the results using invasive technique.
\end{abstract}

\section{Introduction}

The development of science in field of health is very rapid, many studies have explained the correlation between the increase of blood cholesterol levels and the occurrence of cardiovascular diseases, such as arteriosclerosis and hypertension.[1,2].

In 2012, there were 17.5 million deaths and it was predicted to increase up to 23.4 million in 2030 [3, 4], 80\% will be caused by heart attacks and strokes, and more than $75 \%$ will occur in low and middle income countries. Deaths due to cardiovascular disease are around $34 \%$ at the age of 70 years [3]. Excessive protein brought by cholesterol and triglycerides or low-

*Corresponding author: usmanmr4@gmail.com 
density lipoprotein (LDL) that flows in the blood plasma can cause myocardial infarction (heart attack), stroke or peripheral vascular disease.[5, 6]. To prevent this, it is necessary to conduct routine cholesterol check-up.

Device for measuring total cholesterol available in general clinical laboratories is the one with invasive techniques. Invasive technique procedures require blood samples collection which poses a risk of bruising and inflammation. Research using measurement methods with non-invasive techniques have been widely research such as; to estimate total cholesterol with bioelectric impedance [6, 7]. Measurement of total blood cholesterol uses a near-infrared sensor $[4,8]$, using a smart phone camera as a scanner to scan a blood sample in a container to determine total blood cholesterol [9]. This non-invasive method has attracted attention because it is easy to use and inexpensive. At present, there are many individuals who do not pay attention to their cholesterol levels because invasive blood cholesterol monitoring is quite expensive.

Research on non-invasive techniques using near infrared sensors (NIR) to measure blood components including blood glucose, NIR sensors with Emitter LED 1550E, FGA10 photodiode with $800-1800 \mathrm{~nm}$ wavelength [10], wavelength $940 \mathrm{~nm}$ with NIR radiation in $700-1100 \mathrm{~nm}[11,12]$. Measurement of blood cholesterol uses optical sensors IR LED Photodiode wavelength $800 \mathrm{~nm}-1800 \mathrm{~nm}$ [8]. The development of simple measuring devices with non-invasive techniques to monitor total blood cholesterol is required.

Cholesterol has a chemical formula $\mathrm{C} 27 \mathrm{H} 46 \mathrm{O}$, a small biological molecule called lipid such as wax, mixed in blood plasma. In water, cholesterol is almost insoluble $(0.095 \mathrm{mg} / \mathrm{L}$ at $30^{\circ} \mathrm{C}$ ) so that it moves in the bloodstream with a very small concentration [6]. Cholesterol requires lipoproteins to be able to flow in the bloodstream, where hydrophilic proteins attached to cholesterol and formed a lipoprotein. The accumulation of this lipoprotein can cause clogged arteries. Lipoprotein which causes atherosclerosis is called atherogenic. Lipoproteins are classified into Low density lipoprotein (LDL) and High density lipoprotein (HDL) [13]. In 2013, The Ministry of Health of Republic Indonesia referred to the National Cholesterol Education Program - Adult Treatment Panel (NCEP-ATP III) to determine the standard of reference value for total blood cholesterol [14], as shown in table 1 below.

Table 1. Total cholesterol reference value.

\begin{tabular}{|c|c|c|}
\hline Category & LDL $(\mathbf{m g} / \mathbf{d l})$ & Total $(\mathbf{m g} / \mathbf{d l})$ \\
\hline Optimal & $<100$ & \\
\hline Desirable & $100-129$ & \\
\hline $\begin{array}{c}\text { Near optimal/above } \\
\text { optimal }\end{array}$ & $130-159$ & $200-239$ \\
\hline Borderline High & $160-189$ & $>240$ \\
\hline High & $>190$ & \\
\hline Very High & & \\
\hline
\end{tabular}

LDL volume can be determined if the total cholesterol, HDL and triglyceride values are identified, all of which are measured directly in blood $[6,8,13]$ following equation (1). 


$$
L=C-H-k T
$$

$H=$ HDL cholesterol, $L=\mathrm{LDL}$ cholesterol, $C=$ total cholesterol, $T=$ triglycerides and $k$ $=0.20$ if it measured in units of $\mathrm{mg} / \mathrm{dl}$ and $k=0.45$ if it measured in units of $\mathrm{mmol} / \mathrm{l}$.

When radiation interacts with biological tissue, the light is weakened by absorption and scattering, so light attenuation can be described [11]. The description of light propagation in a similar medium as described by the Lambert Beer law is the absorptions of

light at specific wavelengths in homogeneous solution which can be determined accurately using the following equation (2):

$$
I_{X}=I_{O} e^{-\varepsilon(\lambda) \beta L}
$$

Equation (2) $I_{x}$ is the reflected light intensity, $I_{o}$ the incidence of light intensity, $\varepsilon$ is media absorption and media absorption coefficient, $\beta$ is the concentration of absorption and $L$ is the length of the light path. The correlation between light absorbed by the material and transmitted is described by the following equation (3) :

$$
\begin{gathered}
T_{S}=I / I_{O}=10^{-\varepsilon \beta L} \\
A=\varepsilon * \beta * L=\log (T)
\end{gathered}
$$

Where $T_{s}$ is transmittance, $A$ is absorbance. Definition of the reflected light using input voltage Vin and output voltage Vout are substitution of the light intensity and light incident described (4) as described in the following linear equation (4).

$$
\frac{V_{\text {in }}-V_{\text {out }}}{V_{\text {in }}}=10^{-\varepsilon \beta L}
$$

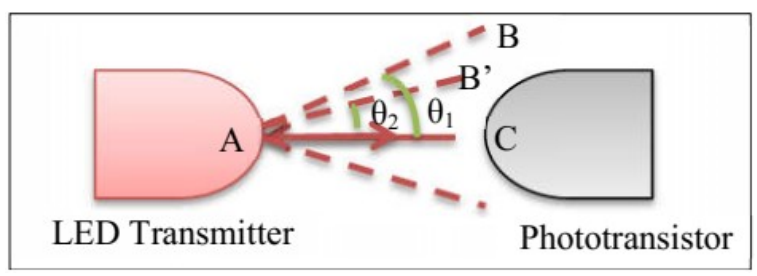

Fig. 1. IR LED light reflection angle.

Determining blood cholesterol concentration is related to the light reflectance angle. Direct correlation between light intensity $J$ and output voltage Vout is described (5). Figure 1 describes the refractive index $\left(\eta_{2}\right)$ to measure cholesterol levels by connecting points $A, B$ and $C$. The increase of cholesterol levels in the blood can reduce the reflection angle $\left(\theta_{2}\right)$, so that the line $\mathrm{AB}$ moves closer to line $\mathrm{AC}$ and creates the new line $\mathrm{AB}$ '. Mathematically, the refractive index $\left(\eta_{2}\right)$ can be calculated using Snell's law. The reflection angle $\left(\theta_{2}\right)$ decreases when the blood cholesterol level increases which reduces the reflected index $\left(\eta_{2}\right)$ and increases output voltage (Vout) because many photons touch the photodiode. As a result 
of the decrease in the reflection angle $\left(\theta_{2}\right)$, the radius of BC in LED light also decreases when light rays curving to AC which causes the increase of cholesterol levels according to Snell's law [12].

$$
\begin{aligned}
V_{\text {out }} & =\frac{n K T}{q} \operatorname{In}\left(\frac{J I_{S C}}{I_{O}}\right)=\frac{n K T}{q}\left[\operatorname{In} \frac{I_{S C}}{I_{O}}+\operatorname{InJ}\right] \\
V_{\text {out }} & =V+\frac{n K T}{q} \operatorname{InJ}
\end{aligned}
$$

Where: $I_{o}$ is Backflow saturation, ISC is Short circuit current, $V$ is Initial voltage $n K T / q=$ is a constant value at $T$ temperature, while $K$ value $=1,380 * 10^{23}$ and $q=1,602^{*} 10^{-19}$.

The transfer of light energy to the material carried by each photon, as described in the following equation (6):

$$
E=h C / \lambda
$$

Where $\mathrm{h}$ is Plank's Constant $\left(6,620^{*} 10^{34} \mathrm{~m} 2 \mathrm{~kg} / \mathrm{s}\right), \mathrm{C}$ is Light Speed $(2,9 * 108 \mathrm{~m} / \mathrm{s})$, and $\lambda$ is the light wavelength $(940 \mathrm{~nm})$.

Equation (6) shows that the amount of energy carried by photons depends on the wavelength where the NIR is at $400-1400 \mathrm{~nm}$, the shorter the wavelength, the higher the wave number and the photon energy.[15].

Real-Time System is a system that must generate the right response and display monitoring within the specified time limit. Real-time system implementation on blood cholesterol total monitoring devices with non-invasive techniques is needed. Blood cholesterol levels are determined by detection of a NIR sensor with a predetermined time limit. When the limit time has been reached the device must display the precise measurement results on the monitor, and users can quickly find out the total blood cholesterol value at that time.

\section{Methodology}

Measurement of blood cholesterol levels with non-invasive techniques was done by conducting blood irradiation on body tissues such as at the earlobe and fingers using the wavelength. Near infrared spectroscopic sensors using infrared LEDs and photodiode was developed to determine blood components such as SPO2, Glucose, Hemoglobin and Heartbeat. Previous research showed good results. Light absorption at wavelengths is utilized by measuring transmission or reflectance. The use of Infrared is a spectroscopy technique employing the infrared wavelength region of an electromagnetic spectrum. Non-invasive techniques analyse absorption ratios at various sets of these wavelengths.

The flow diagram of this study, starting from initialization by converting analog to digital by processing the signals from infrared LED sensors, placing sensors at the fingertips, emitting light into body tissues which then absorbed by the bloodstream and the one not absorbed is reflected and accepted by the photodiode. The voltage from the photodiode is supplied to the ADC microcontroller and calculated using logarithmic equation to determine the total cholesterol value and the readings are displayed on the LCD monitor and sent to the user's smartphone. 


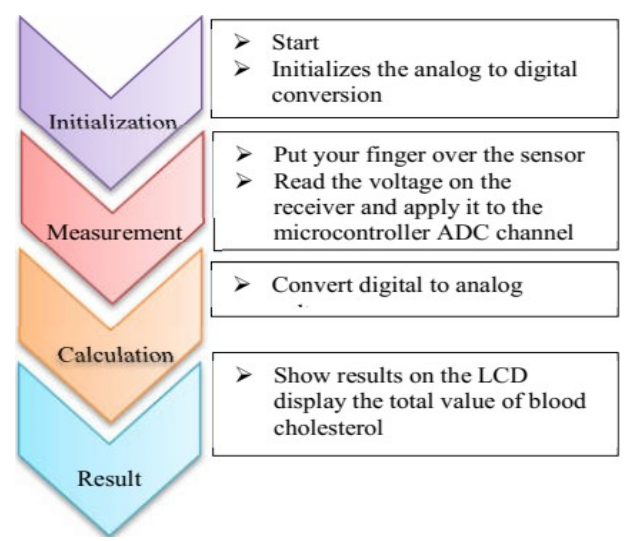

Fig. 2. Diagram of methodology process.

This research used Near Infrared (NIR) sensors with IR LED 333-A Ever light as an emitter with a wavelength of $940 \mathrm{~nm}, 100 \mathrm{~mA}$ continuous current, and $1 \mathrm{~A}$ peak current. The detector used was PT 333-3C Ever light photodiode with a spectral bandwidth of 400nm $1100 \mathrm{~nm}$, and spectral sensitivity at a wavelength of 940nm and maximum voltage of 5 volts. The photodiode absorbed the reflected light emitted by the LED emitter, then converting it into output voltage to the microcontroller. The blood cholesterol monitor device was designed to convert the output voltage into the total cholesterol value in $\mathrm{mg} / \mathrm{dl}$.

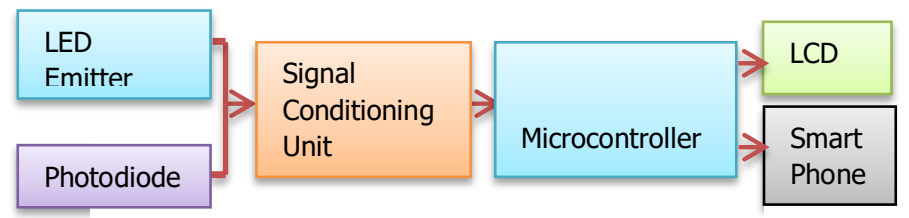

Fig. 3. Diagram block of the blood cholesterol level monitor

Figure 3, a series of sensors consists of IR LED $940 \mathrm{~nm}$ as emitter that emits infrared light. Photodiode is used as a detector that absorbs or receives light. When the sensor is installed at the finger, the light from the LED will be absorbed by the tissue in the finger, and the one not absorbed is reflected and received by the photodiode detector. The light received by the photodiode becomes a light attenuation value and is converted into an electric current, then becomes a voltage due to the presence of a load resistor on the anode. The voltage released by photodiode depends on the amount of light received, the more light is received, the greater the voltage released by the photodiode that is between $0 \mathrm{~V}$ and $5 \mathrm{~V}$. The voltage value of the photodiode is still too low, so the variation in voltage values is less noticeable. To deal with this, an IC LM358N amplifier circuit is installed to strengthen the voltage from the sensor. The voltage value of the photodiode from the sensor circuit is read by a 10-bit ADC microcontroller and this ADC value is reconverted into a voltage value. Then, it is entered into a linear equation to change it into total blood cholesterol value. 


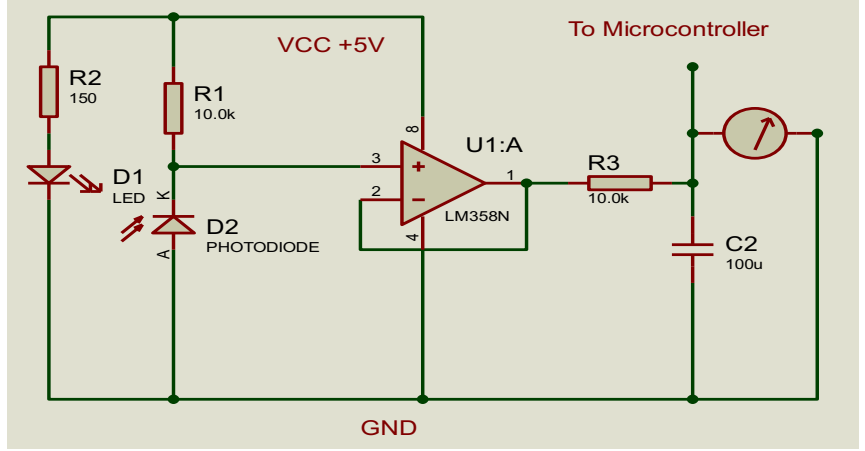

Fig. 4. Transmission Circuit

The measurement results are displayed on the LCD and smartphone. A compatible LCD with a microcontroller and a 4 x 16 LCD screen is used and installed directly on pin 4 on the main board of the microcontroller. The results are sent to smartphone using SIM900A Mini V4.0 Wireless Data Transmission Module GSM GPRS Board Kit installed on the ports available on the main board of the microcontroller.

In order to test the device, a sample was taken from 40 people with ages varied from 20 70 years of male and female participants. Blood cholesterol total data was collected using invasive measuring devices, and then continued with measurement of voltage using the designed NIR sensor a few minutes later. For data collection of sensor voltage output values, each participant was measured five times. The data collection was conducted by measuring the sensor output voltage twice before taking a blood sample for an invasive technique measurement and then thrice afterwards. Sensor output voltage stability, as long as the sensor was on the finger for about 20-40 seconds, the output voltage value was averaged. Data collection techniques and measurement results are shown in Figure 5 and table 2 respectively.
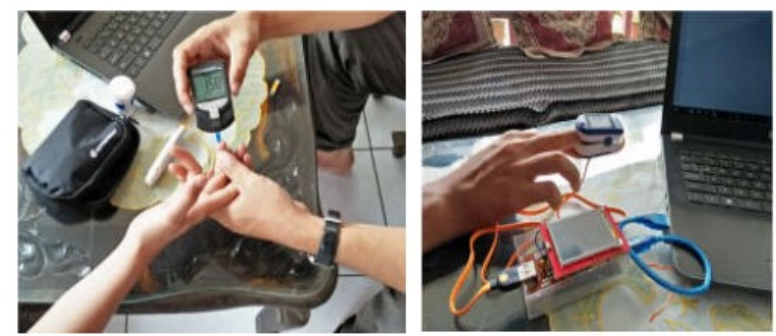

Fig. 5. Data collection with invasive and sensors devices.

Tabel 2. Measurement of the sensor output voltage and total blood cholesterol

\begin{tabular}{|c|c|c|c|c|c|c|}
\hline \multirow{3}{*}{ SUBJECT } & INVASIVE & \multicolumn{5}{|c|}{ NIR SENSOR OUTPUT VOLTAGE } \\
AUTOCHECK & \multicolumn{5}{|c|}{ (Volt) } \\
\cline { 3 - 7 } & $(\mathbf{m g} / \mathbf{d l})$ & SI & SI & NI & NI & NI \\
\hline 1 & 128 & 1.98 & 1.97 & 1.98 & 1.97 & 1.98 \\
\hline
\end{tabular}




\begin{tabular}{|c|c|c|c|c|c|c|}
\hline 2 & 132 & 2.08 & 2.08 & 2.08 & 2.08 & 2.08 \\
\hline 3 & 166 & 2.16 & 2.16 & 2.17 & 2.16 & 2.16 \\
\hline 4 & 226 & 2.25 & 2.28 & 2.27 & 2.26 & 2.25 \\
\hline 5 & 256 & 2.35 & 2.34 & 2.33 & 2.30 & 2.35 \\
\hline 6 & 267 & 2.39 & 2.25 & 2.39 & 2.38 & 2.38 \\
\hline 7 & 290 & 2.42 & 2.43 & 2.43 & 2.43 & 2.43 \\
\hline 8 & 304 & 2.52 & 2.51 & 2.53 & 2.52 & 2.53 \\
\hline 9 & 313 & 2.65 & 2.65 & 2.65 & 2.65 & 2.65 \\
\hline 10 & 350 & 2.70 & 2.71 & 2.71 & 2.70 & 2.70 \\
\hline
\end{tabular}

\section{Result and Discussion}

The results of measurements carried out on male and female participants showed a correlation between sensor voltage output and total cholesterol values, and invasive measurement techniques. The difference in sensor output voltage was found due to the absorption of light in the body's tissues and different light reflections. Blood cholesterol of each person caused this difference. The simple correlation of the total cholesterol values and sensor output voltage with algorithm equation $Y=878.85 \ln (x)-502.81$, with regression correlation coefficients $R^{2}=0.9656$. The NIR sensor output voltage has not been linear with blood cholesterol values measured with invasive techniques. Factors influencing the measurement result include the inconsistent of sensor installation, the possibility of other light received by the photodiode producing impure light reflection and the thickness of the skin affecting the absorption of light in the finger tissue.

In order to determining the total blood cholesterol values showed on the LCD according to the sensor output voltage, a linear equation was needed. Simple data processing with linear regression has the equation $Y=355.87 x-591.46$, with a regression correlation coefficient $R^{2}=0.9747$. The function of this equation was to design a program on a microcontroller. Performance improvements are needed to maintain the stability of the input voltage with the addition of a voltage stabilizer to obtain a high degree of accuracy. 


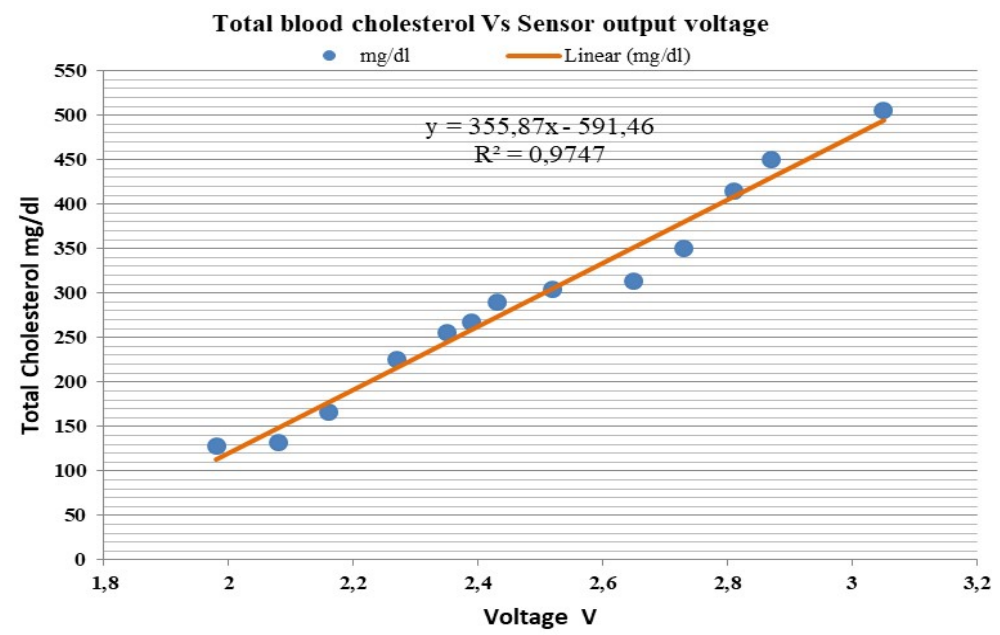

Fig. 6. Linear regression of sensor output voltage and total cholesterol.

Subsequent experiments were conducted to validate by comparing the measurements results of total blood cholesterol obtained using an invasive and a designed non-invasive measuring device. Data obtained using invasive measuring devices is very accurate because it uses blood samples. The results of a non-invasive measuring device depend on the NIR sensor detection. Testing the device by conducting validation was done by measuring total blood cholesterol with non-invasive techniques twice before and after collecting blood samples for invasive techniques.

Table 3. The Measurement Results Using Invasive and Voltage with Non-Invasive Sensors.

\begin{tabular}{|c|c|c|c|c|c|c|}
\hline \multirow{2}{*}{ SUBJECT } & \multicolumn{2}{|c|}{$\begin{array}{c}\text { NON- } \\
\text { INVASIVE } \\
(\mathbf{m g} / \mathbf{d l})\end{array}$} & INVASIVE & \multicolumn{2}{|c|}{$\begin{array}{c}\text { NON- } \\
\text { INVASIVE } \\
(\mathbf{m g} / \mathbf{d l})\end{array}$} & \multicolumn{2}{|c|}{$\begin{array}{c}\text { (mg/dl) } \\
\text { VARIANCE }\end{array}$} \\
\cline { 2 - 6 } & $\mathbf{1}$ & $\mathbf{2}$ & $\mathbf{3}$ & $\mathbf{4}$ & $\mathbf{5}$ & \\
\hline A & 150 & 155 & 158 & 154 & 157 & 3,1 \\
\hline B & 219 & 224 & 226 & 227 & 225 & 3,1 \\
\hline C & 250 & 258 & 252 & 255 & 250 & 3,5 \\
\hline D & 270 & 275 & 267 & 280 & 278 & 5,4 \\
\hline E & 295 & 289 & 290 & 294 & 290 & 2,7 \\
\hline F & 301 & 310 & 306 & 300 & 305 & 4,0 \\
\hline
\end{tabular}

From Table 3, it can be seen that the data differences between the two devices still exist with variants 2,7 to 5,4 . This tolerance value shows a great accuracy, and still needs continuous improvement. The perfect variant is zero, the farther away from zero, the condition of the data is uneven, meaning the greater the value of the variance, the higher the 
fluctuation of data with other data. In subject $\mathrm{E}$, the smallest data variant shows the results relative same between invasive and non-invasive measurements; the accuracy of noninvasive measurements is quite good. Subject D and other subjects showed that the results of non-invasive measurements were still varied; there is high-value data and low-value data from invasive measurements. Factors that influence the measurement results are not evenly distributed on non-invasive techniques such as; finger sensor mounting inconsistent; the sensor is not strong enough to clamp a finger, the possibility of other light entering and being captured by the photodiode and finger thickness can also affect the results of sensor detection.

\section{Conclusion}

The data presented in this paper are results of cholesterol measurements using non-invasive techniques that have been developed using sensors consisting of IR LED emitters and Photodiode detectors. These measurement results were compared with the results taken from invasive technique procedures measurement. The results of this device development are alternative innovations that can be used to measure cholesterol, which are easy to use, not painful, and low cost because it does not use cholesterol strips. The accuracy of the tool has developed quite well and reliable.

\section{Acknowledgment}

The author would like to express his gratitude to Poltekes Muhammadiyah Makassar, Risnawaty Alyah and Syahrir who has provided support and assistance in this research.

\section{References}

1. C. B. Mathews, T. Libish, B. Kaushalkumar, V. Vivek, R. Prabhu, and P. Radhakrishnan, Optoelectronics letters, 12, 1 (2016)

2. M. A. Al-Rawhani et al., IEEE Sensors Journal, 17, 2 (2017)

3. W. H. Organization, Hearts: technical package for cardiovascular disease management in primary health care, (2016)

4. I. Marhaendrajaya, E. Hidayanto, Z. Arifin, and H. Sutanto, Youngster Physics Journal, 6, 3 (2017)

5. S. Yusuf et al., New England Journal of Medicine, 374, 21 (2016)

6. E. Aristovich and S. Khan, Journal of Physics: Conference Series, 450, 1, p. 012057 : IOP Publishing, (2013)

7. G. A. Roth et al., New England Journal of Medicine, 372, 14 (2015)

8. I. Yusoff, R. Yahya, W. Omar, and L. Ku, Innovation \& Commercialization of Medical Electronic Technology Conference (2015)

9. V. Oncescu, M. Mancuso, and D. Erickson, Lab on a Chip, 14, 4, (2014)

10. R. A. Buda and M. M. Addi, Biomedical Engineering and Sciences (IECBES), IEEE Conference (2014)

11. J. Yadav, A. Rani, V. Singh, and B. M. Murari, Biomedical Signal Processing and Control, 18 (2015) 
12. H. Ali, F. Bensaali, and F. Jaber, IEEE Access, 5 (2017)

13. E. Law, M. Kakani, M. Agarwal, and M. Rizkalla, Open Journal of Applied Biosensor, 4, 01 (2017)

14. S. M. Grundy et al., Circulation, 106, 25 (2002)

15. M. NIRSystems, ed Metrohm AG, Herisau (2002) 